Hypoglycemia and the central nervous system. In Symposium on Pediatric Neurology, Ed. Millichap JG, Ped Clin N Amer Nov $1967 ; 14: 865)$. Alternative substrates, particularly ketone bodies, can be used for energy by the brain and may have a protective effect during episodes of hypoglycemia. (Persson B et al. Acta Paediatr Scand 1972;61:273).

\title{
HYPOGLYCEMIA AND BRAIN DAMAGE
}

The neurochemical effects, patterns of cerebral blood flow derangement, time course and distribution of brain tissue damage, and selective neuronal necrosis resulting from hypoglycemia are compared to the biological effects of ischemia and epilepsy - in a neurological progress report from the Departments of Pathology and Clinical Neurosciences, University of Calgary, Alberta, Canada, and the Laboratory for Experimental Brain Research, University Hospital, Lund, Sweden. Hypoglycemia interferes with cerebral energy production, causing membrane depolarization, loss of ion homeostasis, and lipolysis with accumulation of free fatty acids. Tissue aspartate and quinolinic acid levels rise and glutamate levels in CNS tissue fall during hypoglycemia. Cerebral blood flow is increased during the insult and decreased following the insult. Energy failure is moderate in degree with ATP levels dropping to 25 to $30 \%$ of control. Unlike ischemia and epilepsy, hypoglycemia is not accompanied by lactic acidosis. The duration of hypoglycemic insult required to produce selective neuronal necrosis is 10-20 min and neuronal death occurs within 1-8 hrs. (Auer RN, Siesjo BK. Biological differences between ischemia, hypoglycemia, and epilepsy. Ann Neurol Dec 1988;24:699-707).

COMMENT. Ischemia, hypoglycemia, and epilepsy, long thought to produce similar or identical brain damage, apparently have different mechanisms and effects on brain tissue. Insults of equal duration are not equipotent in causing brain damage. Ischemia is much more potent than hypoglycemia, and hypoglycemia is more potent than status epilepticus per minute of insult. The timing of neuronal necrosis evolves over hours to days in ischemia, minutes to hours in hypoglycemia, and during or very shortly after the insult of status epilepticus.

\section{METHYLMALONIC ACIDEMIA AND EXTRAPYRAMIDAL DISEASE}

Four children, 4-13 yrs old, with methylmalonic acidemia who developed acute dystonia after metabolic decompensation with ketoacidosis are reported from the Department of Pediatrics, University of Pennsylvania School of Medicine, and the Children's Hospital of Philadelphia, PA, and other collaborating institutions. The neurological complications were the result of acute necrosis of the globus pallidus and some involvement of internal capsules. One patient, aged 5 yrs, was a poor feeder and had vomited soon after birth. She was hospitalized at 1 wk of age with lethargy and ketoacidosis, and had massive amounts of methylmalonic acid in the urine that were not reduced by IM cyanocobalamin. Treatment with long-term oral alkali, and a 
diet restricted in isoleucine, valine, methionine, and threonine resulted in improvement and normal growth and development up to 2 yrs of age when she had an acute "metabolic stroke" precipitated by otitis media. Subsequently, she developed dystonia, dysarthria, dysphagia, and spastic quadriplegia. A CT scan showed bilateral symmetric lucencies of the globus pallidus and internal capsules. The lesions were thought to result from deranged organic acid metabolism and accumulation of toxic metabolites in the brain. (Heidenreich $R$ et al. Acute extrapyramidal syndrome in methylmalonic acidemia: "Metabolic stroke" involving the globus pallidus. J Pediatr Dec 1988; 113: 1022-7).

COMMENT. Lesions of the basal ganglia have been noted in other inborn errors of organic acid metabolism, including propionic acidemia, and glutaric aciduria type 1, in Leigh syndrome and the mitochondrial encephalomyopathies including Kearns-Sayre and MELAS syndromes, and in several other hereditary diseases involving the CNS.

\section{PEROXISOMAL DISORDERS}

The biochemical and clinical diagnostic characteristics of peroxisomal disorders are reviewed by authors from the Department of Pediatrics, Medical University of South Carolina,

- Charleston, SC. Two major groups are recognized: 1) those that represent a diffuse peroxisomal dysfunction due to (a) a reduction in number of peroxisomes (Zellweger's syndrome, neonatal adrenoleukodystrophy, infantile Refsum's disease, and hyperpipecolic acidemia); and (b) normal numbers but reduced activities of multiple enzymes (rhizomelic chondrodysplasia punctata); and 2) those disorders with specific single peroxisomal enzymatic defects (childhood adrenoleukodystrophy-Xlinked, adult Refsum's disease, hyperoxaluria, acatalasemia, and pseudo-Zellweger's syndrome).

Peroxisomal disorders should be considered in any infant with hypotonia and delays in psychomotor development, and especially in those with facial dysmorphisms (high and prominent forehead), hepatomegaly, cataracts, retinitis, calcific stippling, short limbs, failure to thrive, seizures, and hearing deficit. In childhood, loss of motor skills, progressive dementia, and skin bronzing should suggest the diagnosis. Measurement of very long chain fatty acids is used to confirm the biochemical defect, and other tests including bile acid, phytanic acid, and plasmalogen are included for specific diagnoses. (Singh I et al. Peroxisomal disorders. Biochemical and clinical diagnostic considerations. AJDC Dec 1988; 142:12971301 ).

COMMENT. Peroxisomal disorders are rare but important because clinical sequelae can be related to specific biochemical deficits and some may be identified prenatally and their recurrence prevented. Dietary restriction of very long chain fatty acids and plasmapheresis are helpful 